\title{
PSO-based Optimized Canny Technique for Efficient Boundary Detection in Tamil Sign Language Digital Images
}

\author{
Dr M Krishnaveni ${ }^{1}$ \\ Assistant Professor \\ Department of Computer Science \\ Avinashilingam Institute for Home \\ Science and Higher Education for \\ Women \\ Coimbatore, Tamil Nadu, India
}

\author{
Dr P Subashini $^{2}$ \\ Professor \\ Department of Computer Science \\ Avinashilingam Institute for Home \\ Science and Higher Education for \\ Women \\ Coimbatore, Tamil Nadu, India
}

\author{
TT Dhivyaprabha ${ }^{2}$ \\ Department of Computer Science \\ Avinashilingam Institute for Home \\ Science and Higher Education for \\ Women \\ Coimbatore, Tamil Nadu, India
}

\begin{abstract}
For the hearing impaired, sign language is the most prevailing means of communication for their day-to-day life. It is always a challenge to develop an optimized automated system to recognize and interpret the implication of signs expressed by the hearing impaired. There are a wide range of algorithms developed for SLR, in which only few considerable approaches are carried out in Tamil Sign Language Recognition. This paper has proposed a significant contribution in segmentation process which is the most predominant component of image analysis in constructing the SLR system. Segmentation is handled using edge detection procedure for finding the borders of hand sign within the captured images, by detecting the split in the image illumination. The objective of the edge function, to find the boundary intensity, is done by a particle swarm optimization technique which chooses the optimal threshold values and implemented in the canny hysteresis thresholding method. The analysis primarily uses common edge recognition algorithms which contain Sobel, Robert, Canny and Prewitt from which the scope of the work is extended by introducing an optimization technique in Canny method. The performance of the proposed algorithm is tested with real time Tamil sign language dataset and comparison is inevitably carried out with standard segmentation metrics.
\end{abstract}

Keywords- Tamil Sign Language; Canny Edge Detection; PSO; Thresholding; Objective Function

\section{INTRODUCTION}

Sign language is an inevitable tool for developing understanding skills among deaf children [16]. Exploration of SLR system is very much imperative for hearing impaired people, especially to improve their professional activities. Tamil Sign Language (TSL) is a region-based sign language considered to be a more useful means to have confined improvement in their own boundary [5]. This language is gaining factual importance since it supports an enhanced regional communication. An automated system that identifies and recognizes the TSL signs will be a fine contribution to the regional deaf community.

UGC - BSR Research Start -Up- Grant
Goyal, et al., (2013) proposed a sign language recognition system based on SIFT (scale invariance Fourier transform) to recognize 26 different signs in Indian Sign Language and the feature extraction process had taken place from it to identify the signs distinctly. The outcome of the experimental study shows that the proposed method gives 95\% accuracy for 45 types of input images [15]. Raut, et al., (2015) implemented an Otsu's algorithm for edge detection in Indian Sign Language images. The observed results indicate that the thresholding based segmentation method produces better results in terms of recognition and conversion of different signs into text [10]. Koli, et al., (2015) introduced image processing technique based sign language converter for hearing impaired people and the investigated outcomes state that it gives fairly good results for recognition of different gestures [8]. Archana, et al., (2014) studied vision-based hand gestures used in ISL (Indian Sign Language) interpretation system and reported that implementation of optimization techniques and algorithms could improve the efficiency of identifying various signs in the recognition system [1]. Singha and Das (2013) proposed a sign language recognition system using eigen value weighted euclidean distance for classifying different signs and the examined results illustrate that it achieves $97 \%$ classification accuracy rate for various type of 24 sign images [7].

The related works signify that a wide variety of researches are already existed in the field of Indian Sign Language recognition system. However, developing an SLR system based on evolutionary computation technique and benefaction to Tamil Sign Language recognition are an inattentive area. The main objective of this paper is to propose a major research component in the segmentation stage by using an optimal boundary detection technique with support from computational intelligence. Initially, the image is resized into $256 \times 256$ and converted into grayscale image and that image is preprocessed by optimized weighted median noise filtering technique [9]. The experiments are carried with Tamil letters like 12 Uyir and 1 Aayutha Ezhuthu and each represents the Static images of the palm side of right hand. Figure 4 depicts the generated TSL dataset. The organization of the paper is as 
follows: Section 2 gives the introduction over edge preserving details through thresholding concepts. Section 3 explains the implementation of conventional edge detection methods in real time TSL datasets. Section 4 explains the proposed optimized canny edge detection method using PSO. Section 5 illustrates the results and analysis based on evaluation assessments of the experimental results. Section 6 concludes the findings and analyses the scope of the research work in TSLR.

\section{EDGE DETECTION USING THRESHOLDING}

Edge detection is a process of finding the sharp contrasts in the intensities of an image, by reducing the amount of data in an image, while preserving important structural features of that image [11][14]. Edges can be classified according to their intensity profiles. Edge detection methods depend on the computation of image gradients and the type of filters used to calculate gradient estimates in the horizontal and vertical directions. If the magnitude at a pixel exceeds a threshold, edge point is identified. Therefore, the threshold value is the one which decides whether edges are present or not at an image point [6].This is determined by a single parameter known as the intensity threshold which equals the average value of image pixel intensities. The very importance of it is the understanding of the content of an image and its applications. Finding the edge pixels by thresholding is done after measuring the gradient magnitude of the image. If the threshold is low, it may detect the irrelevant features, and if it is high, it may result in segmented edges. This results in producing thick edges, even if there is a need for thinned segmentation output. The important problem that has to be addressed in this issue is the choosing of thresholds. A commonly used method for finding the appropriate thresholds can be thresholding with hysteresis. However, this problem of choosing appropriate threshold values may vary over the image [12]. This paper deals with the objective of finding the optimal threshold values by implementing nature inspired computing technique.

\section{CONVENTIONAL EDGE DETECTION METHODS}

In general, edge detection is performed using early brought forward algorithms such as gradient-based algorithm and template-based algorithm [14]. These algorithms are experimented and compared with the proposed algorithm to evaluate and find its quality of performance. The edge detection method is performed using three different steps, namely: Noise smoothing, Edge enhancement, and Edge localization.

\section{A. Sobel Edge Detector}

The Sobel operator is a discrete differentiation operator, and detection of edges is done by computing the image intensity function [17]. In this method, the mask seems to be on both $\mathrm{x}$ and $\mathrm{y}$ directions, but the information is combined into one single metric.

\section{B. Roberts Edge Detector}

The Roberts operator performs high spatial gradient which often corresponds to edges. Pixel values at each point in the output represent the estimated absolute magnitude of the spatial gradient of the input image at that point. Masking is done similar to Sobel operator.

$$
\text { Sobel }\left[\begin{array}{lll}
-1 & 0 & 1 \\
-2 & 0 & 2 \\
-1 & 0 & 1
\end{array}\right]\left[\begin{array}{ccc}
1 & 1 & 1 \\
0 & 0 & 0 \\
-1 & -2 & -1
\end{array}\right]
$$

$$
\text { Roberts }\left[\begin{array}{ccc}
0 & 0 & 1 \\
0 & -1 & 0 \\
0 & 0 & 0
\end{array}\right]\left[\begin{array}{ccc}
1 & 0 & 0 \\
0 & -1 & 0 \\
0 & 0 & 0
\end{array}\right]
$$

\section{Prewitt Edge Detector}

Prewitt is an appropriate operator to estimate the magnitude and orientation of an edge from the kernel with the maximum response. In the experiment, the set of kernels is limited to 8 possible orientations. The result produced is very similar to Robert method as the experiment has used the same convolving kernels.

$$
\text { Prewitt }\left[\begin{array}{lll}
-1 & 0 & 1 \\
-1 & 0 & 1 \\
-1 & 0 & 1
\end{array}\right]\left[\begin{array}{ccc}
1 & 1 & 1 \\
0 & 0 & 0 \\
-1 & -1 & -1
\end{array}\right]
$$

\section{Canny Edge Detector}

In this method, the output resultant contains thin edges, and the edge pixels are linked using edge tracking procedure. The problem of finding appropriate thresholds is handled with introducing hysteresis thresholding method [2]. It varies the threshold by tracking the edge once and finding the calculated threshold as the second resultant argument. This method is therefore better at suppressing noise and more likely to detect true weak edges [13]. Therefore, the operator finds its design to be an optimal edge detector. The discrete approximation to Gaussian function is done with $\sigma=1.4$.

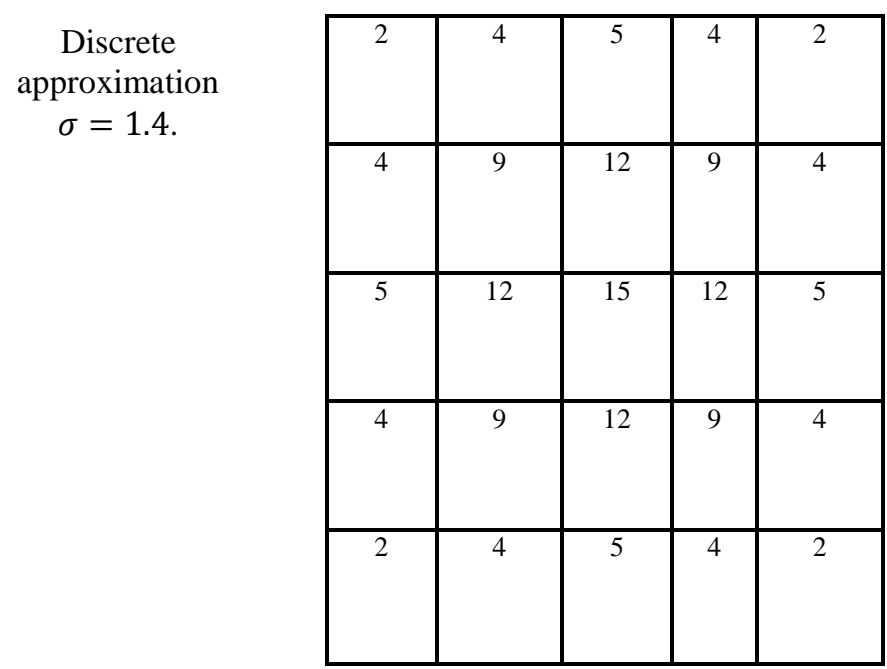

The Sobel operator is performed to find the approximate absolute gradient magnitude at each point with the convolution of mask $\mathrm{G}_{\mathrm{x}}$ and $\mathrm{G}_{\mathrm{y}}$. 


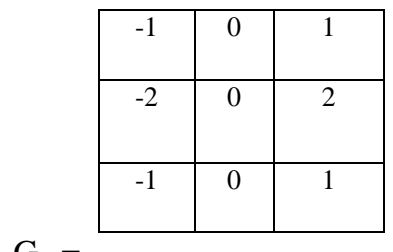

$\mathrm{G}_{\mathrm{x}}=$

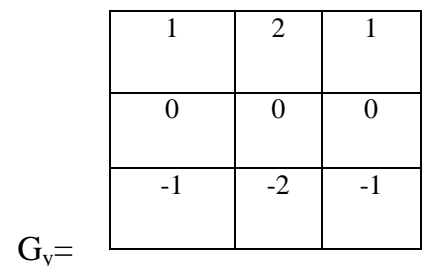

The high threshold value is considered as $\mathrm{H}$ and the low value is named as L. In the image, if the strength of the edge is weaker than the low threshold L, the weak edge is discarded and if the strength of the edge is greater than high threshold $\mathrm{H}$, the true edge is preserved. Similarly, the edge between $\mathrm{L}$ and $\mathrm{H}$ are also kept linked and finds all the edges through connected contours [4]. Figure 1 below depicts the subjective results of the conventional edge detection methods.

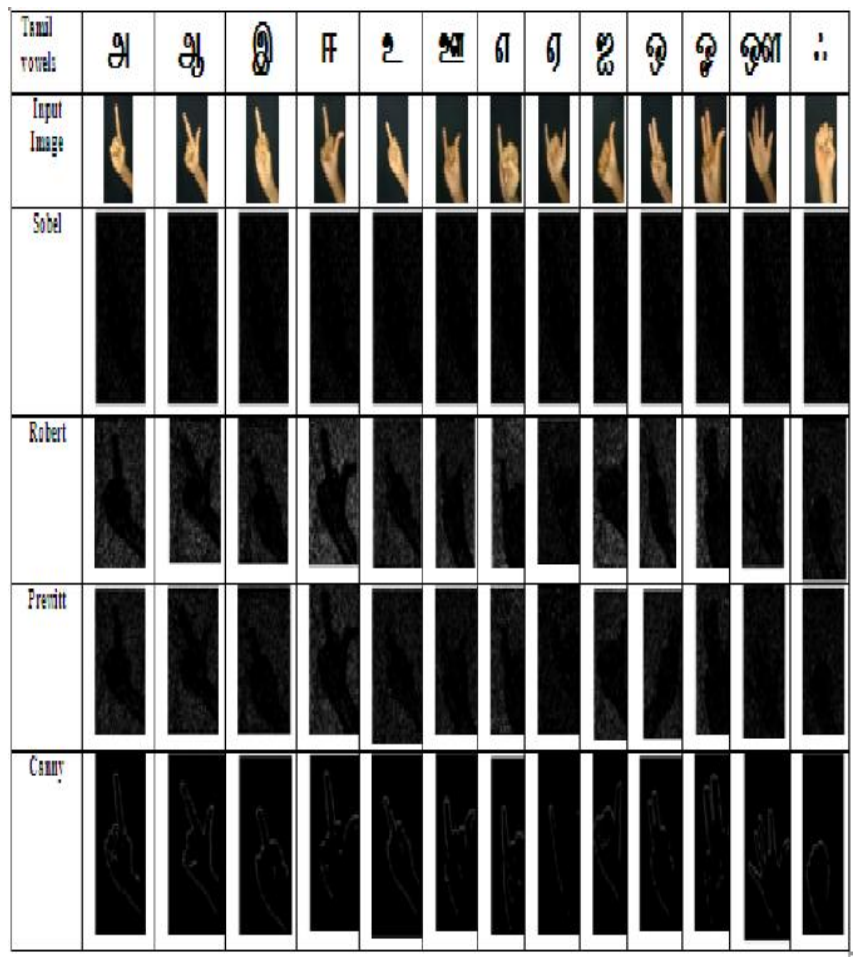

Fig. 1. Visual results of the conventional edge detection methods

\section{PRoposed Optimized CANNY EdGE DETECTION METHOD USING PSO}

Though the appropriate threshold value is chosen in canny method, the edge details that have to be preserved are more and it varies according to the image and its applications. The proposed method defines an objective to choose the optimal threshold values using PSO for implementation of the double thresholding hysteresis method which is a non-linear complex problem [3]. Figure 2 portrays the skeletal view of the canny method optimized using PSO. The particle swarm optimization finds the optimal threshold values (low L and High $\mathrm{H}$ ) for each image and that threshold range is extracted through histogram analysis. The algorithm for the proposed method to find optimal values ( $\mathrm{L}$ and $\mathrm{H})$ is given below:

\section{Proposed AlgorithM:}

Step 1: Input a Tamil sign language image I $(u, v)$ for segmentation.

Step 2: Smoothing - Blurring of the image to remove noise by applying a Gaussian filter. The convolution of an image with a core of Gaussian filter using standard deviation of $\sigma=$ 1.4 is shown in equation (1) given below.

$B=\frac{1}{159}\left[\begin{array}{c}24542 ; 491294 ; 51215125 ; 491294 ; \\ 24542 ;\end{array}\right](1)$

Step 3: Determination of gradients - Find edges by determining gradients of the input image to identify the varying intensity of the image. Gradients at each pixel are found by applying Sobel operator. It can be implemented to approximate the gradient in the $\mathrm{x}^{-}$and $\mathrm{y}$-direction, respectively in the smoothed image by applying the kernels given in Equations (2) and (3):

$G_{x}=\left[\begin{array}{llllll}-1 & 0 & 1 ; & -2 & 0 & 2 ;\end{array}-10-1 ;\right]$

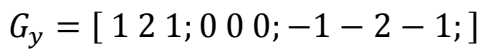

where

$\mathrm{G}_{\mathrm{x}}$ and $\mathrm{G}_{\mathrm{y}}$ are gradient in the $\mathrm{x}$ and $\mathrm{y}$ direction respectively;

The gradient magnitudes can be determined by applying a Euclidean distance measure using the Pythagoras law as shown in equation (4).

$$
|G|=\sqrt{G_{x}^{2}+G_{y}^{2}}
$$

Step 4: Non-maxima suppression $-\mathrm{A}$ gradient image has blurred edges which is transformed into sharp edges by preserving all local maxima and eliminate other pixels in the image. This process consists of the following three steps:

- Round the gradient direction $\theta$ to nearest $45^{\circ}$, corresponding to the use of an 8 connected

neighbourhood pixel values.

- Compare the gradient magnitude of the current pixel with the gradient magnitude of the pixel in the positive and the negative gradient direction.

- If the gradient magnitude of the current pixel is largest, then preserve the value of the edge strength. Otherwise, suppress pixel value in the gradient image.

Step 5: Double thresholding - The resultant edge pixels may still contain noise or irrelevant values. The canny algorithm uses double thresholding (high T1 and low T2) to further suppress the noise content as well as preserve the true image. Edge pixels (T) larger than high threshold (T1) are marked as strong. Edge pixels (T) smaller than low threshold (T2) are removed. Edge pixels (T) falls between T1 and T2 are considered as weak. Selection of threshold values (T1 and T2) can be viewed as a nonlinear complex problem. Here, Particle Swarm Optimization (PSO) algorithm is implemented to choose thresholds for a given sign language image. 
Procedure 1 and Procedure 2 gives the steps to achieve step 5.

Step 6: Edge tracking of image by using hysteresis thresholding - The segmented image is obtained as final output image and it is used for further analysis.

Procedure 1:Steps followed to find the range of threshold values using histogram method: input.

Step 1: A Tamil sign language image dataset is given as

Step 2:The edge of sign image is predetermined as Region of Interest (ROI) in the dataset.

Step 3: Convert the input image into gray scale a image to reduce image size.

\section{Step 4: Repeat}

Identify the histogram $\mathrm{h}(\mathrm{z})$ of an image to be segmented.

Step 5: The probability of a pixel value is represented in the equation (5):

$P(z)=p(z$ /background $) P($ background $)+p($ (zobject $) P$ (object) or

$P(z)=P_{b} \frac{1}{\sqrt{2 \pi \sigma_{b}}} e^{\frac{\left(z-\mu_{b}\right)^{2}}{2 \sigma_{b}^{2}}}+P_{o} \frac{1}{\sqrt{2 \pi \sigma_{o}}} e^{-\frac{\left(z-\mu_{o}\right)^{2}}{2 \sigma_{o}^{2}}} \quad$ or

$P(z)=P_{b} p_{b}(z)+P_{o} p_{o}(z)$

where

$p_{b}(z), p_{o}(z)$ - probability distributions of background and object pixels

$\mu_{b}, \mu_{o}$ - means of the distributions

$\sigma_{b}, \sigma_{o}$ - standard deviations of the distributions

$P_{b}, P_{o}$ - a-priori probabilities of background and object pixels.

Step 6: The probability of misclassification of an object pixel as background is expressed in equation (6):

$E_{o}(T)=\int_{-\infty}^{T} p_{o}(z) d z$

Step 7: The mathematical equation for the probability of incorrectly classifying a background pixel as object is given in (7):

$E_{b}(T)=\int_{T}^{\infty} p_{b}(z) d z$

Step 8: The mathematical formula for threshold selection is obtained by minimizing the above expression as denoted in equation (8):

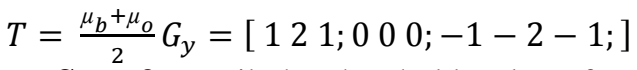

Step 9: Until the threshold values for set of images in Tamil sign language dataset are found.

Procedure2: Steps followed to find optimal threshold values (Low $\mathrm{L}$ and High $\mathrm{H}$ )

Step 1: Initialization of population and parameters - A population of threshold particles says $\mathrm{p}$, of size $\mathrm{m}$ $(0.0<=\mathrm{n}<=1.0)$ are generated and initialized with random position $\mathrm{x}_{\mathrm{p}}$ and velocity $\mathrm{v}_{\mathrm{p}}$.

Step 2: Fitness evaluation of particle - Each particle is evaluated with test function (rotated ellipse2) for choosing the optimal threshold values.
Step 3: Find the individual best particle - Based on the evaluation of fitness function (minima), an individual best (Cognitive) $\mathrm{p}_{\text {best }}$ is chosen for each iteration.

Step 4: Compare the current particle $\left(\mathrm{p}_{\mathrm{i}}\right.$ ) value with the previous value $\mathrm{p}_{\text {besti-1. }}$.

If $\left(\mathrm{pi}<\mathrm{p}_{\text {besti-1 }}\right)$

Else

$$
\text { Set } \mathrm{p}_{\text {best }}=\mathrm{p}_{\mathrm{i}} \text {; }
$$

Set $p_{\text {best. }}=p_{\text {besti-1; }}$

Step 5: Find the social best particle - Identify the neighbourhood (social) particle in the population and assign to index variable $\mathrm{g}$.

$V_{i j}^{(t+1)}=\omega * V_{i j}+\left(c_{1} * r_{1} *\left(P_{\text {best }}-x_{p}\right)+\left(c_{2} * r_{2} *\left(p_{g}-x_{p}\right)\right.\right.$

Step 6: Velocity and position updating equation - The velocity and position of a particle is updated using the following mathematical equations (9) and (10):

$X_{i j}^{(t+l)}=X_{i j}+V_{i j}$

where

$\mathrm{V}_{\mathrm{ij}}=$ velocity of $\mathrm{j}^{\text {th }}$ particle at $\mathrm{i}^{\text {th }}$ iteration

$\mathrm{X}_{\mathrm{ij}}=$ Position of $\mathrm{j}^{\text {th }}$ particle at $\mathrm{i}^{\text {th }}$ iteration

$\omega=0.5-\frac{\operatorname{random}()}{2}$

(i.e) random ( ) function generate a distributed random number which lies from 0 to 1

$\mathrm{c}_{1}=\mathrm{c}_{2}=2.0$

$\mathrm{r}_{1}, \mathrm{r}_{2}=$ random numbers lies between 0 and 1

$\mathrm{P}_{\text {best }}=$ Personal best

$\mathrm{G}_{\text {best }}=$ Neighbourhood (social) particle best

Step 7: Repeat the steps from 3 to 7 for maximum number of iterations.

Step 8: Continuous evolution of swarm in problem space Particle Swarm Optimization (PSO) algorithm offer $\mathrm{P}_{\text {best }}$ and $\mathrm{G}_{\text {best }}$ candidate solution (low and high threshold values) after an evaluation of 5000 iterations.

Step 9: The resultant thresholds (high $\left[\mathrm{T}_{1}\right]$ and low $\left[\mathrm{T}_{2}\right]$ ) are applied for tracking of edges in Tamil sign language image dataset by using hysteresis thresholding.

The subjective evaluation result of canny and optimized canny method using Particle Swarm Optimization is given in Figure 4. 


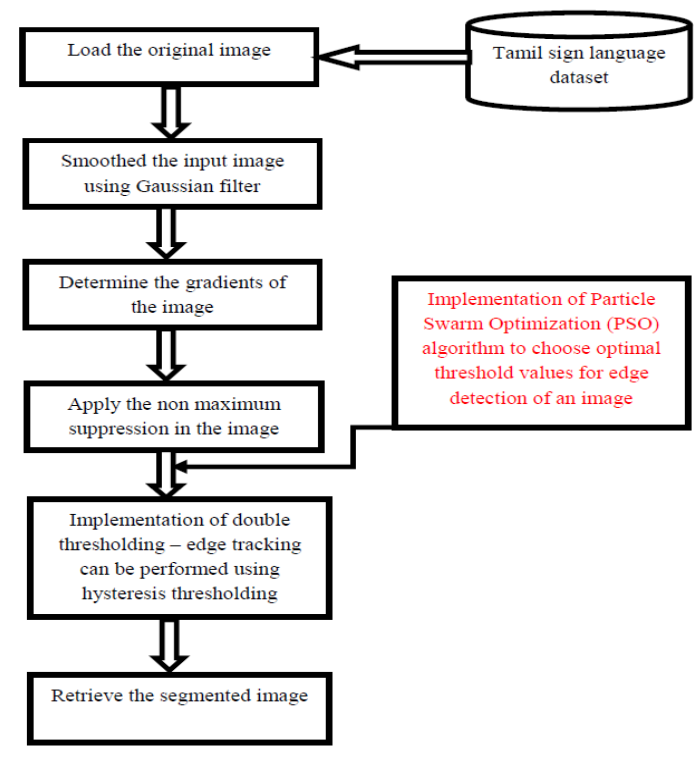

Fig. 2. Skeleton of PSO optimized canny edge detection method for regional sign language images

The canny operator uses a hysteresis thresholding method for detection of edges in the digital image. It uses double threshold values such as $\mathrm{TH}$ (threshold high) and TL (threshold low) where TH > TL. Pixel values greater than TH are classified as edges. The accuracy of the image segmentation process is primarily dependent on threshold value. In this work, the selection of threshold is formulized as a non-linear complex problem. PSO algorithm is implemented to choose global extremum (best solution) stochastically after the fitness evaluation of 5000 runs. The optimal solution retrieved by PSO is applied as threshold values in the canny technique to detect edges in Tamil Sign Language images. The metaheuristic approach is compatible with traditional canny method that gradually improves the probability of attaining the best results in terms of similarity measure. The empirical results shown in Tables I and II signify that the novel method of PSO based canny method produces significant results than conventional edge detectors. Moreover, it is tested with single hand signs but the experimental observation confirmed that the proposed method could perform well for different Tamil signs given by diverse sort of people. The high-quality solution obtained by PSO optimized canny technique is serviceable to hearing impaired people for convey their messages with ordinary people. The goal of this research to reinforce the performance of sign language recognition system by reduces the communication gap between normal people and deaf and dumb people.

\section{RESUltS AND ANALYSIS}

The results are generated by using MATLAB and JAVA. The methodology is experimented with manually generated TSL Datasets with limitation of single handed signs and black background images.
Segmentation of the hand sign is done using edge detection method based on image brightness where it has high discontinuity issues. Though the canny algorithm supports in finding the weak edges using a double threshold method, by minimizing the error rate, identifying process of weaker edge is on the demand of the image characteristics. The performance of the canny algorithm depends on the adjustable parameters like $\sigma$ the standard deviation for Gaussian filter and the two threshold values $\mathrm{TH}$ and $\mathrm{T}_{\mathrm{L}}$. Instead of tailoring the canny algorithm by adjusting the parametric values, optimization technique PSO is introduced to achieve two optimal threshold levels $\mathrm{T}_{\mathrm{h}}$ and $\mathrm{T}_{1 .}$. An example for canny and Nature Inspired Computing [NIC] based optimized canny algorithm is shown in Figure 5 which portrays the edge detected Tamil hand sign language image.

During the execution of PSO, it is terminated after 5000 fitness function evaluations. The particles which are considered to be the range of thresholds are flown and it maintains the diversity in the entire problem space. It slowly converges to give the global optimum solution. Therefore, this nature of PSO is taken to produce optimal thresholding values which are implemented in canny algorithm to improve the quality of edge detection results. The quality of the proposed algorithm can be clearly observed from the metric evaluation in which similarity index and Pearson correlation coefficients values are higher for the proposed method than other conventional methods. From the experiments conducted on TSL datasets, it is inferred that the proposed optimized canny algorithm using PSO gives better results than existing traditional algorithms.

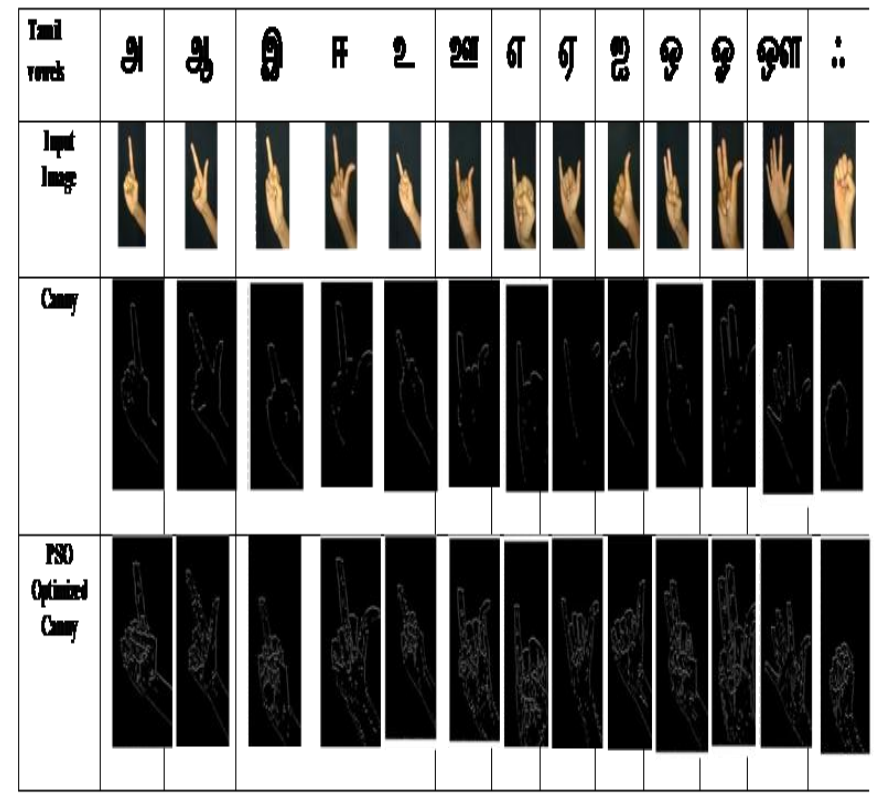

Fig. 3. Visual assessment of Canny and optimized canny algorithm 
TABLE I. OBJECtive Evaluation of the Proposed Method With CONVEnTIONAL METhods Using Similarity INDEX METRIC

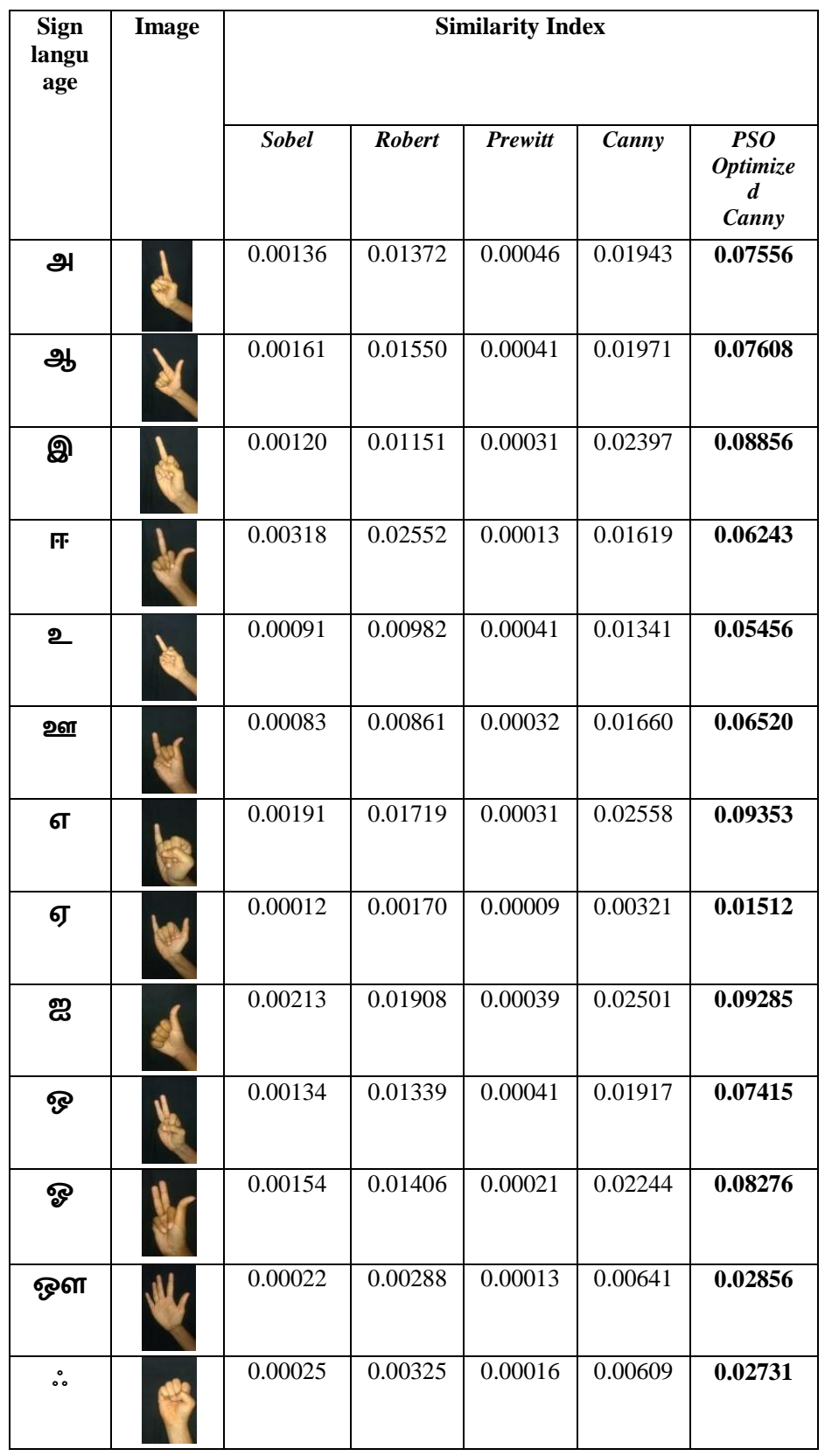

TABLE II. Objective Evaluation of the Proposed Method with CONVENTIONAL METHOdS USING PEARson CORRELATION COEFFICIENT METRIC

\begin{tabular}{|c|c|c|c|c|c|c|}
\hline & Image & \multicolumn{5}{|c|}{ Pearson Correlation Coefficient } \\
\hline $\begin{array}{c}\text { Ayuth } \\
\text { a } \\
\text { Eluthu } \\
\text { kkal }\end{array}$ & & Sobel & Robert & Prewitt & Canny & $\begin{array}{c}\text { PSO } \\
\text { Optimi } \\
\text { zed } \\
\text { Canny }\end{array}$ \\
\hline அ & & -0.0501 & -0.1467 & -0.1095 & 0.0747 & 0.1832 \\
\hline ஆ & & -0.0481 & -0.1468 & -0.1103 & 0.0719 & 0.1458 \\
\hline இ & & -0.0646 & -0.1848 & -0.1378 & 0.0443 & $\mathbf{0 . 1 5 3 7}$ \\
\hline Гా & & -0.0684 & -0.0237 & -0.1514 & 0.0455 & 0.1326 \\
\hline 2 & & -0.0440 & -0.1317 & -0.0979 & 0.0633 & 0.1734 \\
\hline ஊ & & -0.0490 & -0.1495 & -0.1119 & 0.0407 & 0.1660 \\
\hline எ & & -0.0603 & -0.1823 & -0.1364 & 0.0350 & 0.1708 \\
\hline ஏ & & -0.0336 & -0.1042 & -0.0771 & 0.0380 & 0.1351 \\
\hline ஐ & & -0.0563 & -0.1652 & -0.1232 & 0.0519 & 0.1444 \\
\hline ஒ & & -0.0515 & -0.1528 & -0.1140 & 0.0556 & 0.1828 \\
\hline ஓ & & -0.0631 & -0.1826 & -0.1367 & 0.0295 & 0.1329 \\
\hline ஒள & & -0.0373 & -0.1097 & -0.0824 & 0.0548 & 0.1398 \\
\hline$\therefore$ & & -0.0374 & -0.1123 & -0.0847 & 0.0462 & 0.1865 \\
\hline
\end{tabular}

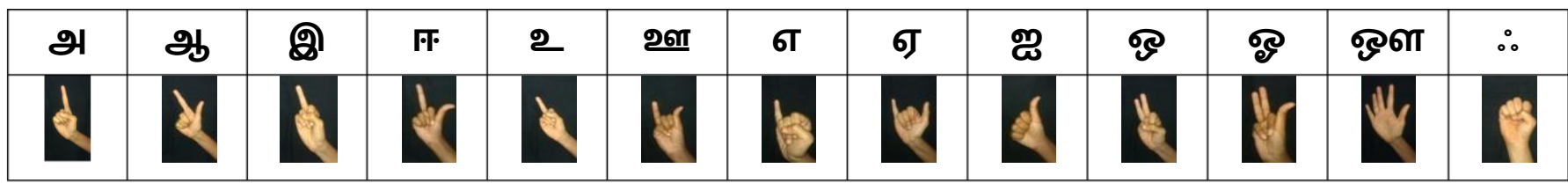

Fig. 4. Manually Generated Tamil Sign Language Dataset (12 Uyir and 1 Aayutha Ezhuthu) 


\section{CONCLUSION}

The main objective of the paper is to develop an optimized edge detector to reduce the number of broken edges and to increase the localization accuracy of edge detection in real time digital images. The goal was successfully achieved by using PSO for optimal search of threshold values for the canny edge detector algorithm. The objective and subjective results show that the new algorithm performs better than the canny and conventional edge detection algorithms. The particle swarm optimization involves rotated ellipse 2 as its objective function which is analyzed to be the best in producing the thin edge results. Experimental results validate its potential detection of weak edges while preserving the fine details of the image. The increase in the value of similarity index and Pearson Correlation Co-efficient across a wide experimental dataset reveals the same as the visual quality. In future, the work can be extended to deeply identify the finger tip detection for TSL recognition system and also to attempt multi-dimensional datasets. In the future, this study will be extended on video based sign language recognition system with dynamic signs and body gestures as well and more work has to be done in the area of feature extraction and classification process.

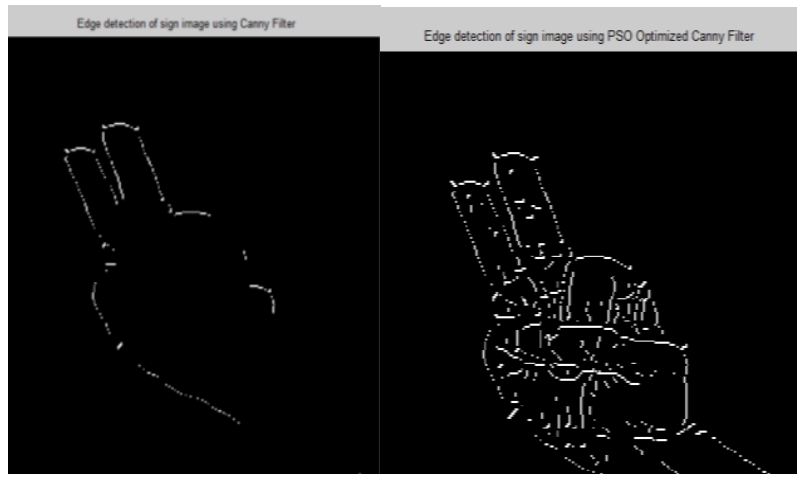

Fig. 5. Edge detected image of a hand sign image before and after optimization of canny algorithm

\section{ACKNOWLEDGMENT}

This work is supported by the project entitled "Investigation on view based methodological approaches for Tamil Sign language Recognition" (No. F. 30-42/2014 (BSR)) funded by UGC - BSR Research Start -Up- Grant.

\section{REFERENCES}

[1] S. ArchanaGhotkar and K. GajananKharate, "Study of vision based hand gesture recognition using indian sign language," International journal on smart sensing and intelligent systems, vol. 7, pp. 96-115, 2014.

[2] J. Canny, "A computational approach to edge detection," IEEE Transaction on Pattern Analysis and Machine Intelligence, vol. 8, pp. 679-698, 1986.
[3] G. Coath, and S. Halgamuge, "A comparison of constraint-handling methods for the application of particle swarm optimization to constrained nonlinear optimization problems," IEEE Congress on Evolutionary Computation, vol. 4, pp.2419-2425, 2003.

[4] Dongjiang $\mathrm{Xu}$, and TakisKasparis, "Detection and localization of edge contours," Geo-Spatial and Temporal Image and Data Exploitation III, Proceedings of SPIE, vol. 5097, pp.79-89, 2003.

[5] P. Ghate, S. Sunder, A. Maricar, and S. Bhatnager, "An introduction to the signing system for indian languages," Part II - Additional Signs. Bombay: Ali Yavar Jung National Institute for the Hearing Handicapped, 1990.

[6] S. Jansi, andP. Subashini, "Optimized adaptive thresholding based edge detection method for mri brain images," International Journal of Computer Applications, vol. 51, pp. 1-8, 2012.

[7] JoyeetaSingha and Karen Das, "Indian sign language recognition using eigen value weighted euclidean distance based classification technique," International Journal of Advanced Computer Science and Applications(IJACSA), vol.4 ,pp. 188-195.

[8] P.B.Koli, Chaudhari Ashwini, MalkarSonam, PawaleKavitaandTaydeAmrapali, "Image processing based language converter for deaf and dumb people," IOSR Journal of Electronics and Communication Engineering (IOSR-JECE), vol. 5, pp. 25-30. 2015.

[9] M. Krishnaveni, P. Subashini, and T.T. Dhivyaprabha, "Efficient removal of impulse noise in tamil sign language digital images using pso based weighted median filter," International Journal of Applied Engineering Research (IJAER), vol. 10, pp. 40474-40480, 2015.

[10] D. ManishaRaut, PallaviDhok, KetanMachhale, and JaspreetManjeetHora, "A system for recognition of indian sign language for deaf people using otsu's algorithm," International Research Journal of Engineering and Technology (IRJET), vol. 2, pp. 228-232, 2015.

[11] Nassir Salman,"Image segmentation based on watershed and edge detection techniques," The International Arab Journal of Information Technology, vol. 3, pp. 104-110, 2006.

[12] H. Pan, L. Wang, and B. Liu, "Particle swarm optimization for function optimization in noisy environment," Journal of Applied Mathematics and Computation, vol. 181, pp. 908-919, 2006.

[13] L. Philip Worthington, "Enhanced canny edge detection usingcurvature consistency," IEEE Transactions on Graphics and Image Processing, vol. 55, pp. 95-105, 2002.

[14] Raman Maini, and Himanshu Aggarwal, "Study and comparison of various image edge detection techniques," International Journal of Image Processing (IJIP), vol. 3, pp. 1-10, 2010.

[15] SakshiGoyal, Ishita Sharma, and Shanu Sharma, "Sign language recognition system for deaf and dumb people," International Journal of Engineering Research \& Technology (IJERT), vol. 2, pp. 382-387, 2013.

[16] TirthankarDasgupta, Sambit Shukla Sandeep Kumar, SynnyDiwakar, and AnupamBasu, "A multilingual multimedia indian sign language dictionary tool," The 6th Workshop on Asian Language Resources, pp. 57-64, 2008.

[17] Wenshuo Gao, Lei Yang, Xiaoguang Zhang, and HuizhongLiu, “An improved sobel edge detection," vol. 10, pp. 67-71, 2010. 